\title{
Exponential and power-law probability distributions of wealth and income in the United Kingdom and the United States
}

\author{
Adrian Drăgulescu, Victor M. Yakovenko*,1 \\ Department of Physics, Center for Superconductivity Research, University of Maryland, \\ College Park, MD 20742-4111, USA
}

\begin{abstract}
We present the data on wealth and income distributions in the United Kingdom, as well as on the income distributions in the individual states of the USA. In all of these data, we find that the great majority of population is described by an exponential distribution, whereas the high-end tail follows a power law. The distributions are characterized by a dimensional scale analogous to temperature. The values of temperature are determined for the UK and the USA, as well as for the individual states of the USA. (C) 2001 Elsevier Science B.V. All rights reserved.
\end{abstract}

PACS: 87.23.Ge; 89.90.+n; 02.50.-r

Keywords: Econophysics; Wealth; Income; Boltzmann; Gibbs; Pareto

\section{Introduction}

The study of wealth and income distributions has a long history. Pareto [1] proposed in 1897 that wealth and income distributions obey universal power laws. Subsequent studies have often disputed this conjecture (see a systematic survey in the World Bank research publication [2]). Mandelbrot [3] proposed that the Pareto law applies only asymptotically to the high ends of the distributions. Many researchers tried to deduce the Pareto law from a theory of stochastic processes. Gibrat [4] proposed in 1931 that income and wealth are governed by multiplicative random processes, which result

\footnotetext{
* Corresponding author. Tel.: +1-301-405-6151; fax: +1-301-314-9465.

E-mail address: yakovenk@physics.umd.edu (V.M. Yakovenko).

${ }^{1}$ http://www2.physics.umd.edu/ yakovenk/
} 
in a log-normal distribution. These ideas were later followed, among many others, by Montroll and Shlesinger [5]. However, already in 1945 Kalecki [6] pointed out that the log-normal distribution is not stationary, because its width increases in time. Modern econophysicists [7-10] also use various versions of multiplicative random processes in theoretical modeling of wealth and income distributions.

Unfortunately, numerous recent papers on this subject do very little or no comparison at all with real statistical data, much of which is widely available these days on the Internet. In order to fill this gap, we analyzed the data on income distribution in the United States (US) from the Bureau of Census and the Internal Revenue Service (IRS) in Ref. [11]. We found that the individual income of about $95 \%$ of population is described by the exponential law. The exponential law, also known in physics as the Boltzmann-Gibbs distribution, is characteristic for a conserved variable, such as energy. In Ref. [12], we argued that, because money (cash) is conserved, the probability distribution of money should be exponential. Wealth can increase or decrease by itself, but money can only be transferred from one agent to another. So, wealth is not conserved, whereas money is. The difference is the same as the difference between unrealized and realized capital gains in stock market.

Unfortunately, we were not able to find data on the distribution of money. On the other hand, we found data on wealth distribution in the United Kingdom (UK), which are presented in this paper. Also presented are the income distribution data for the UK and for the individual states of the USA. In all of these data, we find that the great majority of population is described by an exponential distribution, whereas the high-end tail follows a power law.

\section{Wealth distribution in the United Kingdom}

In this section, we discuss the cumulative probability distribution of wealth $N(w)=$ (the number of people whose individual wealth is greater than $w$ )/(the total number of people). A plot of $N$ vs. $w$ is equivalent to a plot of person's rank in wealth vs. wealth, which is often used for top reach people [13]. We will use the power law, $N(w) \propto 1 / w^{\alpha}$, and the exponential law $N(w) \propto \exp (-w / W)$, to fit the data. These distributions are characterized by the exponent $\alpha$ and the "temperature" $W$. The corresponding probability densities, $P(w)=-\mathrm{d} N(w) / \mathrm{d} w$, also follow a power law or an exponential law. For the exponential law, it is also useful to define the temperatures $W^{(2)}$ (also known as the median) and $W^{(10)}$ using the bases of $\frac{1}{2}$ and $\frac{1}{10}: N(w) \propto$ $\left(\frac{1}{2}\right)^{w / W^{(2)}} \propto\left(\frac{1}{10}\right)^{w / W^{(10)}}$.

The distribution of wealth is not easy to measure, because people do not report their total wealth routinely. However, when a person dies, all assets must be reported for the purpose of inheritance tax. Using these data and an adjustment procedure, the British tax agency, the Inland Revenue (IR), reconstructed wealth distribution of the whole UK population. In Fig. 1, we present the 1996 data obtained from their Web site [14]. The left panel shows the cumulative probability as a function of the personal total net 

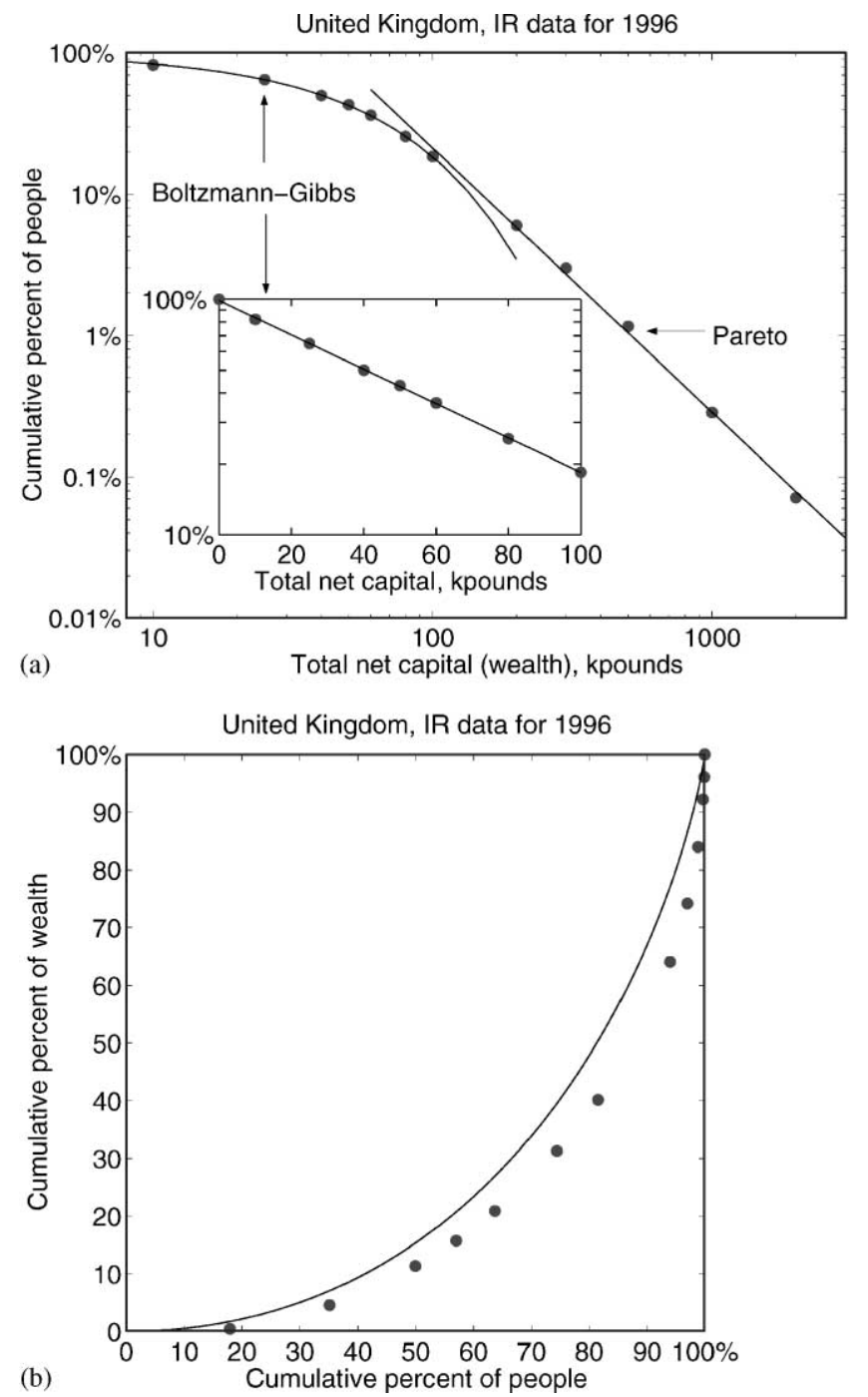

Fig. 1. Cumulative probability distribution of total net capital (wealth) shown in $\log -\log$ (a), log-linear (inset), and Lorenz (b) coordinates. Points: the actual data. Solid lines: left panel-fits to the exponential (Boltzmann-Gibbs) and power (Pareto) laws, right panel—function (2) calculated for the exponential law.

capital (wealth), which is composed of assets (cash, stocks, property, household goods, etc.) and liabilities (mortgages and other debts). The main panel illustrates in the log$\log$ scale that above $100 \mathrm{k} £$ the data follow a power law with the exponent $\alpha=1.9$. The inset shows in the $\log$-linear scale that below $100 \mathrm{k} £$ the data is very well fitted by an exponential distribution with the temperature $W_{U K}=59.6 \mathrm{k} £\left(W_{U K}^{(2)}=41.3 \mathrm{k} £\right.$ and $W_{U K}^{(10)}=137.2 \mathrm{k} £$ ).

The right panel of Fig. 1 shows the so-called Lorenz curve [2,11] for wealth distribution. The horizontal and vertical coordinates are the cumulative population $x(w)$ and 
the cumulative wealth $y(w)$ :

$$
x(w)=\int_{0}^{w} P\left(w^{\prime}\right) \mathrm{d} w^{\prime}, \quad y(w)=\int_{0}^{w} w^{\prime} P\left(w^{\prime}\right) \mathrm{d} w^{\prime} / \int_{0}^{\infty} w^{\prime} P\left(w^{\prime}\right) \mathrm{d} w^{\prime} .
$$

The points represent the actual data, whereas the solid line is calculated for a purely exponential distribution [11]:

$$
y=x+(1-x) \ln (1-x) .
$$

One can see that the data systematically deviate from the exponential law because of the wealth concentrated in the power-law tail; however, the deviation is not very big. The so-called Gini coefficient [2,11], which measures the inequality of wealth distribution, has increased from $64 \%$ in 1984 to $68 \%$ in 1996 [14]. This value is bigger than the Gini coefficient $50 \%$ for a purely exponential distribution [11]. The inequality of the US income distribution was also increasing during that time period [11].

\section{Income distribution in the United Kingdom}

We obtained the data on the yearly income distribution in the UK for $1997 / 1998$ and 1998/1999 from the Web site of the IR [15]. The data for 1994/1995, 1995/1996, and 1996/1997 were taken from the Annual Abstract of Statistics derived from the IR [16]. The data for these 5 years are presented graphically in Fig. 2. In the upper inset of the left panel, the original raw data for the cumulative distribution are plotted in $\log$-linear scale. For not too high incomes, the points form straight lines, which implies the exponential distribution $N(r) \propto \exp (-r / R)$, where $r$ stands for income (revenue), and $R$ is the income "temperature". However, the slopes of these lines are different for different years. The temperatures for the years 1994/1995, 1995/1996, 1996/1997, and $1997 / 1998$ differ from the temperature for $1998 / 1999, R_{U K}^{(98 / 99)}=11.7 \mathrm{k} £\left(R_{U K}^{(2)}=8.1 \mathrm{k} £\right.$ and $R_{U K}^{(10)}=26.9 \mathrm{k} f$ ), by the factors $0.903,0.935,0.954$, and 0.943 . To compensate for this effect, we rescale the data. We divide the horizontal coordinates (incomes) of the data sets for different years by the quoted above factors and plot the results in $\log -\log$ scale in the main panel and log-linear scale in the lower inset. We observe scaling: the collapse of points on a single curve. Thus, the distributions $N_{i}(r)$ for different years $i$ are described by a single function $f\left(r / R_{i}\right)$. The main panel shows that this scaling function $f$ follows a power law with the exponent $\alpha=2.0-2.3$ at high incomes. The lower inset shows that $f$ has an exponential form for about $95 \%$ of individuals with lower incomes. These results qualitatively agree with a similar study by Cranshaw [17]. He proposed that the $P(r)$ data for lower incomes are better fitted by the Gamma distribution $\Gamma(r) \propto r^{\beta} \exp (-r / R)$. For simplicity, we chose not to introduce the additional fitting parameter $\beta$.

We must mention that the individuals with income below a certain threshold are not required to report to the IR. That is why the data in the lower inset do not extend to zero income. We extrapolate the straight line to zero income and take the intercept 

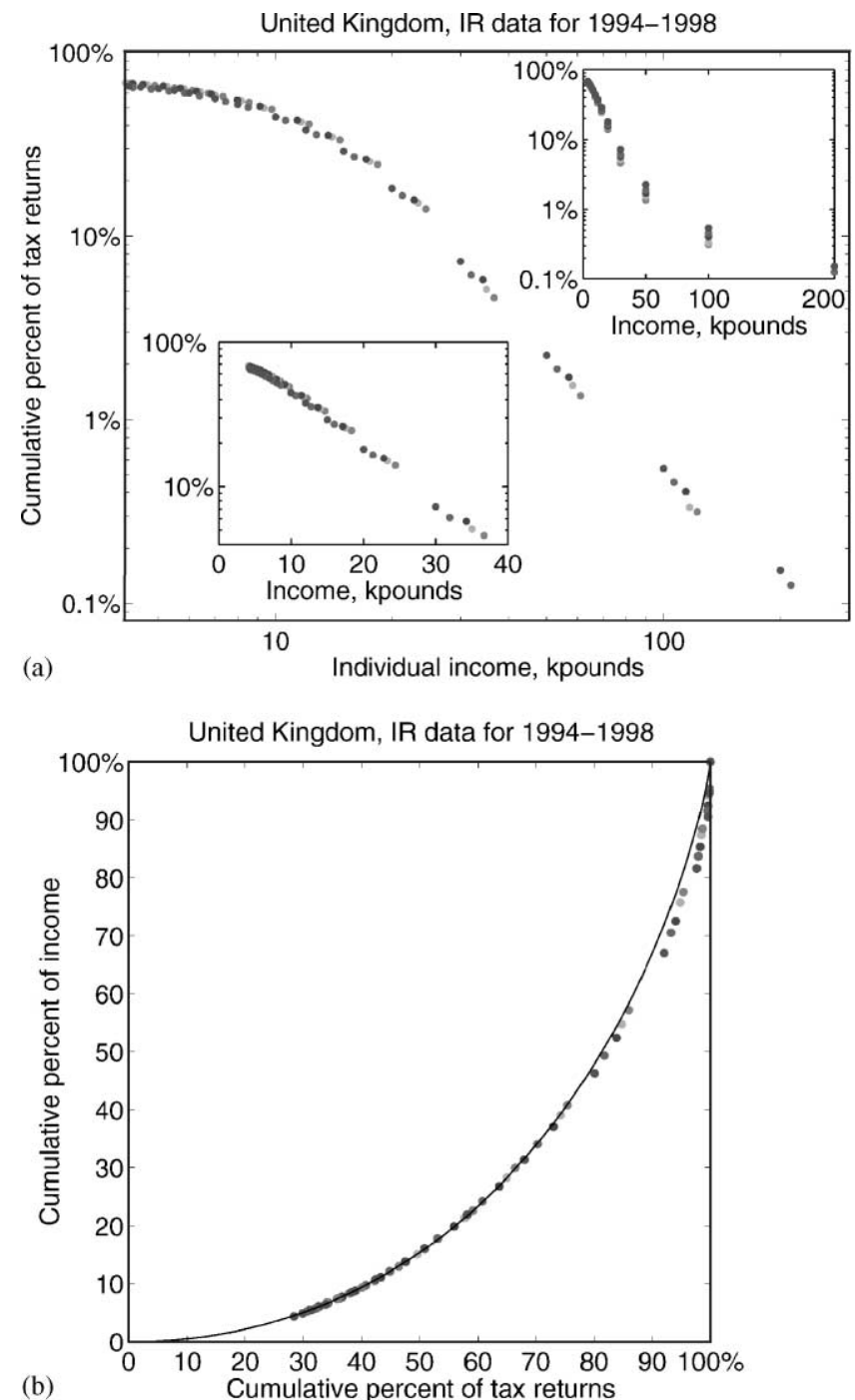

Fig. 2. Cumulative probability distributions of yearly individual income in the UK shown as raw data (top inset) and scaled data in $\log -\log$ (a), log-linear (lower inset), and Lorenz (b) coordinates. Solid curve: fit to function (2) calculated for the exponential law.

with the vertical axis as $100 \%$ of individuals. Thus, we imply that the IR data does not account for about $25-27 \%$ of individuals with income below the threshold.

The Lorenz curve for the distribution of the UK income is shown in the right panel of Fig. 2. We treat the number of individuals below the income threshold and their total income as adjustable parameters, which are the horizontal and vertical offsets of the coordinates origin relative to the lowest known data point. These parameters are chosen to fit the Lorenz curve for the exponential law (2) shown as the solid line. The 
fit is very good. The horizontal offsets are $28-34 \%$, which is roughly consistent with the numbers quoted for the lower inset of the left panel.

\section{Income distribution in the United States}

We obtained the data on distribution of the yearly individual income in 1998 for each of the 50 states and the District of Columbia that constitute the USA from the Web site of the IRS [18]. We plot the original raw data for the cumulative distribution of income in log-linear scale in the upper inset of the left panel of Fig. 3. The points spread significantly, particularly at higher incomes. For example, the fraction of individuals with income greater than $1 \mathrm{M} \$$ varies by an order of magnitude between different states. However, after we rescale the data in the manner described in the preceding section, the points collapse on a single curve shown in log-log scale in the main panel and loglinear scale in the lower inset. The open circles represent the US average, obtained by treating the combined data for all states as a single set. We observe that the distribution of higher incomes approximately follows a power law with the exponent $\alpha=1.7 \pm 0.1$, where the \pm 0.1 variation includes $70 \%$ of all states. On the other hand, for about $95 \%$ of individuals with lower incomes, the distribution follows an exponential law with the average US temperature $R_{U S}=36.4 \mathrm{k} \$\left(R_{U S}^{(2)}=25.3 \mathrm{k} \$\right.$ and $\left.R_{U S}^{(10)}=83.9 \mathrm{k} \$\right)$. The temperatures of the individual states differ from $R_{U S}$ by the amounts shown in Table 1. For example, the temperature of Connecticut (CT) is 1.25 times higher and the temperature of West Virginia (WV) is 0.78 times lower than the average US temperature.

The Lorenz plot for all states is shown in the right panel of Fig. 3 together with the solid curve representing Eq. (2). The majority of points are well clustered and are not too far from the solid curve. The exceptions are Wyoming (WY) with much higher inequality and the Washington state (WA) with noticeably lower inequality of income distribution. The average US data, shown by open circles, is consistent with our previous results [11]. Unlike in the UK case, we did not make any adjustment in the US case for individuals with income below the threshold, which appears to be sufficiently low.

\section{Discussion}

We found scaling in the cumulative probability distributions $N(r)$ of individual income $r$ derived from the tax statistics for different years in the UK and for different states in the US. The distributions $N_{i}(r)$ have the scaling form $f\left(r / R_{i}\right)$, where the scale $R_{i}$ (the temperature) varies from one data set $i$ to another, but the scaling function $f$ does not. The function $f$ has an exponential (Boltzmann-Gibbs) form at the low end, which covers about $95 \%$ of individuals. At the high end, it follows a power (Pareto) law with the exponents about 2.1 for the UK and 1.7 for the US. Wealth distribution 

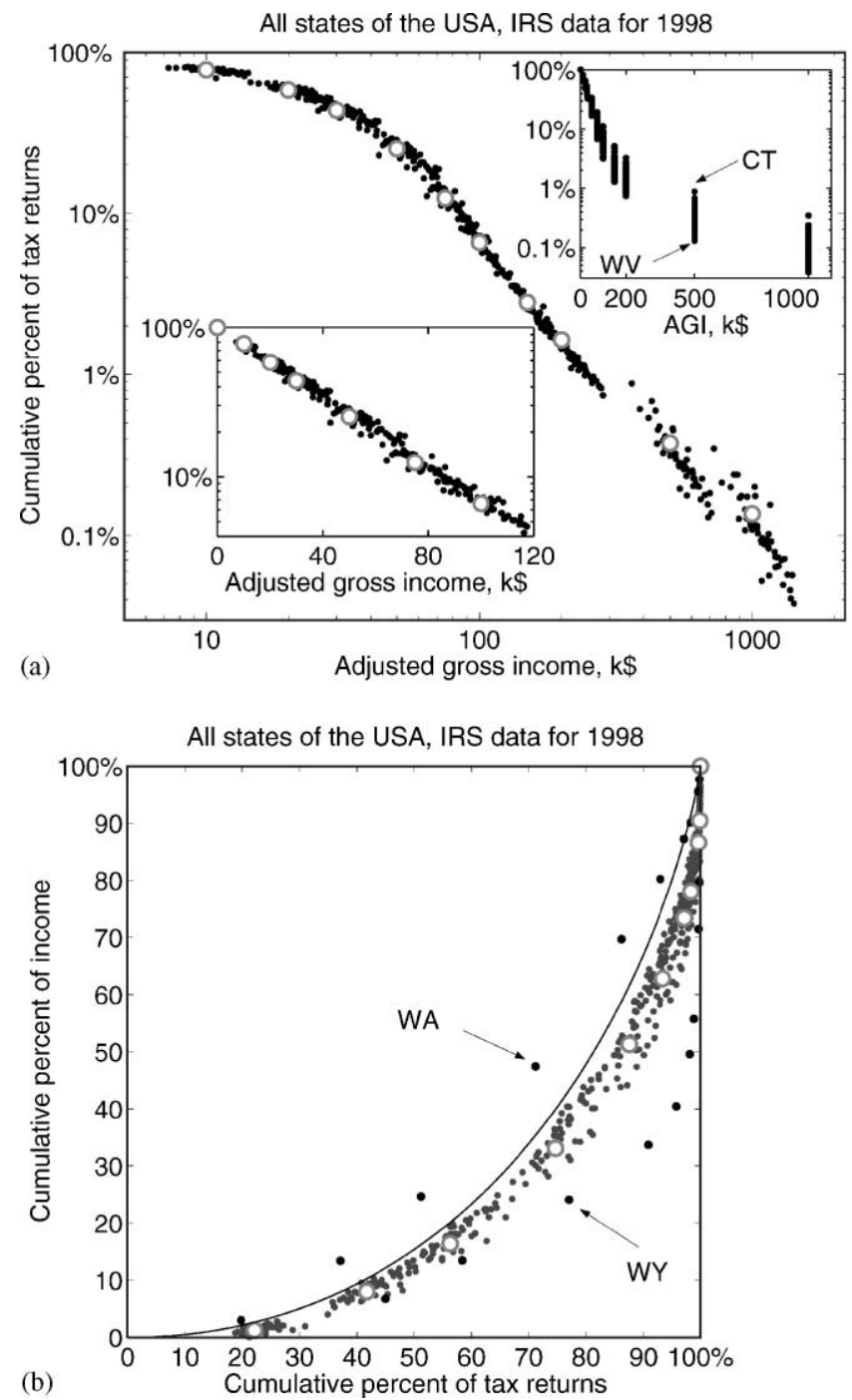

Fig. 3. Cumulative probability distributions of yearly individual income for different states of the USA shown as raw data (top inset) and scaled data in $\log -\log$ (a), log-linear (lower inset), and Lorenz (b) coordinates. Solid curve: function (2) calculated for the exponential law.

Table 1

Deviations of the state temperatures from the average US temperature

\begin{tabular}{lllllllllllllllllll}
\hline CT & NJ & MA & MD & VA & CA & NY & IL & CO & NH & AK & DC & DE & MI & WA & MN & GA \\
$25 \%$ & $24 \%$ & $14 \%$ & $14 \%$ & $9 \%$ & $9 \%$ & $7 \%$ & $6 \%$ & $6 \%$ & $5 \%$ & $5 \%$ & $5 \%$ & $4 \%$ & $4 \%$ & $2 \%$ & $1 \%$ & $0 \%$ & \\
TX & RI & AZ & PA & FL & KS & OR & HI & NV & NC & WI & IN & UT & MO & VT & TN & NE \\
$-1 \%$ & $-3 \%$ & $-3 \%$ & $-3 \%$ & $-4 \%$ & $-5 \%$ & $-6 \%$ & $-7 \%$ & $-7 \%$ & $-7 \%$ & $-8 \%$ & $-8 \%$ & $-9 \%$ & $-9 \%$ & $-9 \%$ & $-11 \%$ & $-12 \%$ \\
OH & LA & AL & SC & IA & WY & NM & KY & ID & OK & ME & MT & AR & SD & ND & MS & WV \\
$-12 \%$ & $-13 \%$ & $-13 \%$ & $-13 \%$ & $-14 \%$ & $-14 \%$ & $-14 \%$ & $-14 \%$ & $-15 \%$ & $-16 \%$ & $-16 \%$ & $-19 \%$ & $-19 \%$ & $-20 \%$ & $-20 \%$ & $-21 \%$ & $-22 \%$ \\
\end{tabular}


in the UK also has a qualitatively similar shape with the exponent about 1.9 and the temperature $W_{U K}=60 \mathrm{k} £$. Some of the other values of the exponents found in literature are 1.5 proposed by Pareto himself $(\alpha=1.5), 1.36$ found by Levy and Solomon [13] for the distribution of wealth in the Forbes 400 list, and 2.05 found by Souma [19] for the high end of income distribution in Japan. The latter study is similar to our work in the sense that it also uses tax statistics and explores the whole range of incomes, not just the high end. Souma [19] finds that the probability density $P(r)$ at lower incomes follows a log-normal law with a maximum at a nonzero income. This is in contrast to our results, which suggest that the maximum of $P(r)$ is at zero income. The discrepancy may be due to the high threshold for tax reporting in Japan, which distorts the data at the low end. On the other hand, if the data is indeed valid, it may reflect the actual difference between the social structures of the US/UK and Japan.

The income temperature for the UK in $1998 / 1999$ was $R_{U K}=11.7 \mathrm{k} £$ and for the US in 1998 was $R_{U S}=36.4 \mathrm{k} \$$. Using the exchange rate as of December 31,1998 to convert pounds into dollars [20], we find that the UK temperature was $R_{U K}=19.5 \mathrm{k} \$$, which is 1.87 times lower than the US temperature. The difference in temperatures indicates nonequilibrium, which can be exploited to create a thermal machine [12]. The gain (profit) produced by such a thermal machine is proportional to the difference in temperatures. In agreement with the second law of thermodynamics, money would flow from a high-temperature system to a low-temperature one. This may explain the huge trade deficit of the USA in global international trade with other, lower-temperature countries. The variation of temperatures between different states of the USA is shown in Table 1.

\section{Acknowledgements}

We are grateful to Ted Cranshaw, who kindly sent us his unpublished study of income distribution in the UK [17] and data [16], and Bertrand Roehner, who suggested to study income distributions in the states of the USA and sent us link [18].

\section{References}

[1] V. Pareto, Cours d'Economie Politique, Lausanne, 1897.

[2] N. Kakwani, Income Inequality and Poverty, Oxford University Press, Oxford, 1980.

[3] B. Mandelbrot, Int. Econom. Rev. 1 (1960) 79.

[4] R. Gibrat, Les Inégalités Economique, Sirely, Paris, 1931.

[5] E.W. Montroll, M.F. Shlesinger, J. Stat. Phys. 32 (1983) 209.

[6] M. Kalecki, Econometrica 13 (1945) 161.

[7] Zhi-Feng Huang, S. Solomon, Physica A 294 (2001) 503.

[8] S. Solomon, P. Richmond, cond-mat/0102423, Physica A 299 (2001) 188-197 [these proceedings].

[9] J.-P. Bouchaud, M. Mezard, Physica A 282 (2000) 536.

[10] D. Sornette, R. Cont, J. Phys. I (France) 7 (1997) 431.

[11] A. Drăgulescu, V.M. Yakovenko, Eur. Phys. J. B 20 (2001) 585.

[12] A. Drăgulescu, V.M. Yakovenko, Eur. Phys. J. B 17 (2000) 723. 
[13] M. Levy, S. Solomon, Physica A 242 (1997) 90.

[14] Distribution of Personal Wealth, Inland Revenue, http://www.inlandrevenue.gov.uk/stats/ distribution2000.pdf.

[15] Personal Incomes, Inland Revenue, 1998-99: Table 3.3 of http:/www.inlandrevenue.gov.uk/stats/ income2000.pdf, 1997-98: http://www.inlandrevenue.gov.uk/stats/table3_3a.htm.

[16] Annual Abstract of Statistics, Office of National Statistics, http://www.statistics.gov.uk.

[17] T. Cranshaw, Pareto, Poverty and Riches, unpublished.

[18] Individual Tax Statistics by States in 1998, IRS, http://www.irs.gov/tax_stats/soi/ind_st.html, file 98IN54CM.EXE.

[19] W. Souma, cond-mat/0011373.

[20] The Interactive Currency Table, http://www.xe.net/ict/. 